\title{
Revisión bibliográfica sobre inclusión financiera como estrategia de recuperación y de crecimiento fintech ${ }^{*}$
}

\author{
Recibido: 31 de agosto de 2020 - Aprobado: 25 de octubre de 2020 \\ https://doi.org/10.22395/seec.v23n55a8
}

Daniel Cardona Valencia**

RESUMEN

El presente artículo pretende dar un acercamiento a la inclusión financiera digital desde la visión postCOVID-19 como posible herramienta de desarrollo y resiliencia. Mediante un seguimiento bibliográfico de análisis institucionales, investigaciones académicas e informes de entidades relevantes, se hace un mapeamiento de los aportes y recomendaciones sobre la temática en materia de recuperación económica. En los resultados se confirma la trascendencia de la inclusión financiera digital como elemento aportante a la reconstrucción y fortalecimiento del flujo económico y del tejido financiero, y se proponen acciones para su correcta adopción.

\section{PALABRAS CLAVE}

Instituciones Financieras, Educación Financiera, Decisiones Financieras, Fintech, Inclusión Financiera.

\section{CLASIFICACIONES JEL}

G23, G32, G41

\section{CONTENIDO}

Introducción; 1. Antecedentes; 2. Metodología; 3. Resultados; 4. Conclusiones y Discusiones; Bibliografía.

El presente artículo es el resultado de la investigación desarrollado en el marco del proyecto de Educación Financiera adscrito al Grupo de Investigación Ciencias Administrativas (Clasificado en categoría A en Colciencias en el año 2020) del Instituto Tecnológico Metropolitano ITM y realizado entre junio y agosto de 2020

.* Ingeniero Industrial y Magister en Finanzas, Universidad de Antioquia, Medellín, Colombia. Especialista en Finanzas y Mercados de Capitales, Universidad de Medellín, Medellín, Colombia. Estudiante de Doctorado en Desarrollo Local y Cooperación Internacional, Universidad de Valencia, Valencia, España. Dirección: Calle 54A No. 30 - 01 Medellín Colombia, Tel: (+574) 46007 27. Correo electrónico: danielcardona@itm.edu.co. Orcid: https://orcid.org/0000-0001-8689-4399 


\title{
LITERATURE REVIEW ON FINANCIAL INCLUSION AS A RECOVERY AND GROWTH STRATEGY FINTECH
}

\begin{abstract}
This article aims to provide an approach to digital financial inclusion based on the post-COVID-19 vision as a potential tool for development and resilience. Based on a bibliographic follow-up of institutional analyses, academic research and reports from relevant entities, a mapping of the contributions and recommendations about economic recovery is made. The results confirm the importance of digital financial inclusion as a contributor to the reconstruction and strengthening of the financial system, and actions for proper adoption are suggested.
\end{abstract}

\section{KEY WORDS}

Financial Institutions, Financial Literacy, Financial Decisions, Fintech, Financial Inclusion

\section{CONTENIDO}

Introduction; 1. background; 2. methodology; 3 . results; 4. conclusions and discussions; Bibliography.

\section{REVISÃO LITERÁRIA SOBRE INCLUSÃO FINANCEIRA COMO ESTRATÉGIA PARA ESTRATÉGIA DE RECUPERAÇÃO E CRESCIMENTO DA FINTECH}

\section{RESUMO}

Este artigo visa proporcionar uma abordagem da inclusão financeira digital a partir da visão pós-fintech como uma ferramenta possível para o desenvolvimento e a resiliência. COVID-19 como uma ferramenta possível para o desenvolvimento e a resiliência. Através de um acompanhamento bibliográfico das instituições de análise institucional, pesquisas acadêmicas e relatórios de entidades relevantes, um mapeamento das contribuições para mapeamento das contribuições e recomendações sobre o tema em termos de recuperação econômica. recuperação. Os resultados confirmam a importância da inclusão financeira digital como um elemento que contribui para a reconstrução e o fortalecimento da economia. e o fortalecimento do fluxo econômico e do tecido financeiro, e propõe ações para sua correta adoção.

\section{PALAVRAS-CHAVE}

Instituições Financeiras, Educação Financeira, Decisões Financeiras, Fintech, Inclusão Financeira.

\section{CLASSIFICAÇÕES DE JEL}

G23, G32, G41

\section{CONTEÚDO}

Introdução; 1. antecedentes; 2. metodologia; 3. resultados; 4. Conclusões e Discussões; Bibliografia. 


\section{INTRODUCCIÓN}

La situación de la COVID-19 ha generado impactos y grandes costos en innumerables ámbitos, destacándose el costo en vidas humanas. Los investigadores de diferentes áreas se han volcado a explicar cómo se tradujo una crisis sanitaria en una crisis económica y la forma en que generó estancamientos en la economía mundial, desde el distanciamiento social que desencadenó en cierres de mercados, hasta la incertidumbre del alcance de la situación en importantes factores como el consumo y la inversión (Ozili y Arun, 2020).

De acuerdo con Naciones Unidas (2020) esta pandemia tiene alcance y dimensiones económicas que representan una amenaza real en el logro de los Objetivos de Desarrollo Sostenible (ODS), una vez que se tiene un gran impacto en diferentes aspectos financieros tales como los costos para el sistema de salud, el incremento de las dificultades alimentarias, el impacto en el turismo y en la inversión extranjera directa, y de manera particular en los países que presentan mayores riesgos.

Los Objetivos de Desarrollo Sostenible fueron propuestos desde el 2015 por Naciones Unidas; antes de la COVID-19 se identificaban retrasos en su cumplimiento y hay indicios de que los efectos de esta pandemia generarán impactos mayores que anteriores crisis (Behravesh y Rocha, 2020). Estos objetivos plantean el alcance de 17 metas para el planeta al año 2030, en relación con sostenibilidad medio ambiental, social y económica (Villa et al., 2020).

Frente a estos alcances, la misma ONU, presenta un plan orientado a derrotar el virus y construir un mundo mejor en el cual además de las amenazas, se identifica la necesidad de solidaridad, de apoyo financiero y se destacan puntos positivos de la crisis, tales como mayor proyección de temas fundamentales para el desarrollo global: el incremento de las Fintech (tecnologías al servicio de las finanzas) y el aumento de la Inclusión financiera (United Nations, 2020). La inclusión financiera se refiere al acceso y uso de los servicios financieros bajo regulaciones que protejan y promulgue el conocimiento en gestión del dinero por parte de los consumidores, con el fin de mejorar el desempeño financiero de toda una población (Raccanello et al., 2014).

Por su parte, las Fintech son aquellas startups de tecnologías financieras que, como propuesta de valor, mejoran la oferta de servicios financieros tradicionales gracias a la generación de experiencias personales a través de canales digitales que generan costos menores y operaciones más amigables (Romanova y Kudinska, 2016).La tecnología en el sector financiero ha sido un aspecto en exploración en los últimos diez años; durante la pandemia de la COVID-19 se han generado más 
oportunidades para que la penetración bancaria y el uso de los servicios financieros digitales se aceleren a causa del distanciamiento social y las medidas restrictivas que limitaron el acceso a la banca tradicional (Sahay et al., 2020).

Estos procesos Fintech ya venían siendo parte de estrategias fundamentales de expansión en los sistemas financieros globales, una vez que, a través del internet, la big data y la seguridad informática, se ha pretendido extender, personalizar y acelerar los servicios financieros (Igual Molina, 2019). La modificación de dichos servicios y su migración a la nube ya se hacía fundamental como estrategia de expansión bancaria y como herramienta de desarrollo (Díaz et al., 2018).

Según el Banco Mundial (2018) a cierre del 2017, 1.7 billones de adultos no estaban bancarizados y no contaban con una cuenta de transacciones básicas por temas como asequibilidad, costos, falta de entidades proveedoras, falta de documentos y falta de confianza en los proveedores de servicios financieros existentes. Por su parte, la situación de aislamiento y disminución de la interacción física incentiva las transacciones sin contacto y el uso de dinero virtual, las cuales son características de despliegue eficiente y rápido, no solo de las finanzas personales sino también de las medidas de apoyo gubernamentales y la inclusión de pequeños negocios en la llamada economía digital (Zhang et al., 2020).

Sahay et al. (2020) sugieren que la inclusión financiera digital debería ser tenida en cuenta en las apuestas de desarrollo en una etapa de recuperación, ya que podría jugar un papel importante en la mitigación del impacto económico y social de la actual crisis de la COVID-19, una vez que la ampliación del acceso financiero de las pequeñas y medianas empresas y de los hogares en condiciones menos favorables podría apoyar a una recuperación generalizada y más inclusiva (Ashraf, 2020).

Desde este documento, se pretende dar un acercamiento a la probable dirección de los efectos de la COVID-19 en términos de desarrollo socioeconómico; a partir de la revisión de análisis académicos, investigaciones y observaciones de entidades internacionales sobre el futuro de la economía mundial y las estrategias propuestas para garantizar el papel fundamental de la inclusión financiera como herramienta de recuperación y de crecimiento. Este artículo de seguimiento bibliométrico cuenta con cuatro partes: un acercamiento a la temática a partir de los antecedentes y evolución de la inclusión financiera desde las posibilidades que da la digitalización de las finanzas a través de las Fintech; una descripción metodológica donde se describe en que consiste un estudio bibliométrico; los resultados obtenidos donde se presentan aprendizajes y premisas de la temática; y, finalmente, conclusiones 
y recomendaciones direccionadas al alcance de la inclusión financiera en la era post-COVID-19.

\section{ANTECEDENTES}

\subsection{Inclusión financiera digital o inclusión financiera posibilitada por la tecnología financiera}

El Fondo Monetario Internacional (2014) propone el termino de inclusión financiera digital o inclusión posibilitada por la tecnología financiera, para referirse al acceso digital y uso de servicios financieros formales, a través de un teléfono móvil y/o computadora. Dentro de este concepto se incluyen los servicios prestados por empresas de tecnología e instituciones financieras. Autores como Ozili (2018) señalan que las finanzas digitales cubren una magnitud no solo de productos, sino también de softwares y negocios relacionados con las finanzas y formas novedosas de comunicarse con los clientes, permitiendo a particulares y empresas tener acceso a servicios de pago, ahorro y crédito sin la necesidad de tener un contacto cercano con el proveedor, en nuevos y mayores espacios y con mejores tiempos de atención. De manera ideal, para referirse a servicios financieros digitales se relaciona una plataforma transaccional, agentes intermediarios y el uso por parte de los clientes a partir de un dispositivo electrónico (Gabor y Brooks, 2017).

El objetivo que tienen estos servicios financieros, al estar disponibles en plataformas digitales tiene dos visiones. Desde las Naciones Unidas y las entidades en pro del desarrollo, su direccionamiento es hacia la reducción de la pobreza y la inclusión financiera de las economías en desarrollo (Naciones Unidas, 2014) y desde la visión general de los mercados y las entidades financieras; su objetivo es hacia la competitividad y expansión de los servicios financieros y el impulso de la productividad en los países, generando estabilidad económica y aumento en la intermediación financiera (Dorfleitner et al., 2017). De acuerdo con Ozili (2018) si en efecto se quiere hablar de inclusión financiera como herramienta de desarrollo y apropiación Fintech, se debe hacer sinergia en estas visiones y ver el potencial desde la accesibilidad y asequibilidad de los servicios financieros mediante sistemas formales y seguros que sí impulsen el desarrollo y generen rentabilidades a largo plazo, pero que también generen mejores tomas de decisiones financieras, bienestar empresarial y social mediante costos nulos o bajos.

La inclusión financiera digital se reconoce como una herramienta que posibilita e impulsa el desarrollo y la recuperación económica de los países, una vez que permite ampliar el alcance del sistema financiero y posibilita también el acceso a apoyos estatales y formalización de la economía (Venkatesh et al., 2016). Un ejemplo de 
éxito es el experimento en la región de Bengala occidental (región donde se ubica la ciudad de Calcuta en la India), donde desde la administración local se implementó entre 2007-2010 como plan de gobierno, una política de divulgación del sector bancario y de generación de préstamos y microcréditos generalizados en zonas rurales y urbanas; la cual generó efectos positivos en la inclusión financiera y en la reducción de la pobreza de los pobladores, los cuales pudieron desarrollar proyectos productivos y mejorar sus condiciones socioeconómicas; aunque también reveló que se deben aplicar medidas desde la oferta y desde la demanda, y diferenciarlas en sectores rurales y urbanos (Chattopadhyay, 2011).

\subsection{Crecimiento de las Fintech}

Con la llegada de la cuarta revolución industrial y la posibilidad ampliada de generación y almacenamiento de información en red, aparecen miles de startups que desafían todos los productos que la banca tradicional podía ofrecer e incursionan en dimensiones que van desde los pagos personales hasta los seguros y el trading (ver figura 1). Todas estas dimensiones del Fintech tienen en común y como identidad, la posibilidad de la interacción hombre-máquina y la reducción de la interacción hombre-hombre (International Monetary Fund, 2014; Rizwan et al., 2020).

\section{Figura 1. Servicios Financieros afectados por innovaciones Fintech}

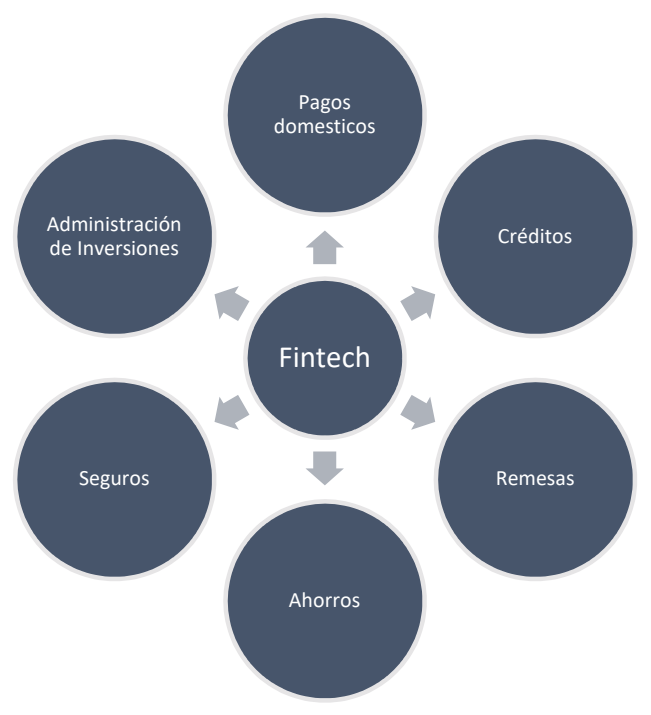

Fuente: Tomado de FMI (2014) 
Estas startups forman el denominado sector Fintech que plantea un modelo donde se desarrollan productos enfocados en la experiencia del cliente y en costos bajos (Igual Molina, 2019). Luego de la crisis de 2007 el malestar y la desconfianza respecto al sistema financiero tradicional y la percepción de cambio necesario, ha logrado que las startups Fintech encuentren financiación por parte de capitales de riesgos y que al año 2019 ya haya sobrepasado los ocho mil millones de dólares (Philippon y Philippon, 2019).

Las apuestas porque el Fintech se masifique han estado en la agenda de muchos líderes mundiales y formuladores de políticas públicas desde mucho antes de la crisis de la COVID-19 (Akhtaruzzaman et al., 2020). La Alianza para la inclusión Financiera (AFI) elabora políticas en este sentido desde el 2008, justo después de la crisis de los subprime (GPFI, 2011). De la misma manera los líderes del G20, desde el 2010 en su cumbre en Seúl propusieron un plan de acción centrado en el desarrollo Fintech y la inclusión financiera $(G 20,2011)$. Adicionalmente, las Naciones Unidas en los ODS plantean de la necesidad de incluir en las agendas nacionales la expansión de los servicios financieros como herramienta en contra de la pobreza (Villa et al., 2020).

Desde el 2018, el Fondo Monetario Internacional y el Banco Mundial lanzaron la agenda Fintech donde se establecen los principios seguros de Fintech y se identifican las principales tendencias en la materia. En esta agenda nace el Índice de Adopción Global Fintech, que busca analizar el crecimiento de las tecnologías financieras y presentar las adopciones y tendencias tecnológicas y de consumo que están siendo implementadas. En la figura 2 se presenta la evolución de las dimensiones Fintech entre el año 2015 y 2019, en donde se destaca el acelerado crecimiento de las tecnologías al servicio de las transacciones cotidianas de dinero como pagos y transferencias, pasando de un $18 \%$ a un $75 \%$ en tan solo 4 años. Por otro lado, se identifica la lenta aplicación de las Fintech en temas como presupuestos, seguros y créditos.

Figura 2. Adopción de categorías Fintech entre 2015 y 2019 en el mundo.

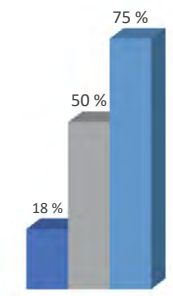

Transferencia de dinero y pagos

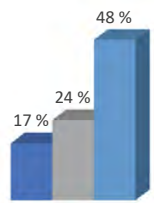

Ahorro e inversion



Presupuestos y planeación financiera



Seguros
Créditos

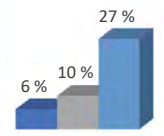

[ $2015=2017 \quad 2019$ 
Las Fintech están cambiando la forma en que se prestan los servicios financieros a las pequeñas empresas y a los hogares de bajos ingresos (Arner et al., 2020). De manera tradicional, los servicios financieros han sido prestados por bancos y sus agentes, instituciones de micro financiación y sistemas informales y ahora a través de los alcances del internet, la big data, el blockchain y la inteligencia artificial; los servicios financieros buscan acercarse a sus clientes e interactuar con ellos de nuevas maneras relacionadas con el uso de celulares, computadores y aplicaciones que generan mayor conectividad y seguimiento en tiempo real a las operaciones (Philippon y Philippon, 2019).

\section{METODOLOGÍA}

El documento presenta un estudio de carácter descriptivo a través de un análisis de bibliografía donde se identifica literatura relevante sobre Inclusión Financiera y Fintech como estrategias de recuperación o desarrollo postpandemia en bases de datos indexadas incluyendo informes y reportes de organismos de cooperación, entidades reconocidas en ámbitos financieros y de desarrollo, y autores que expongan posiciones y reflexiones con relación a la temática para tener un panorama de los posibles direccionamientos de la temática como herramienta de inclusión post-COVID-19.

Para el desarrollo, se utiliza como fuente de información de Scopus ${ }^{\circledR}$ y Google Scholar ${ }^{\circledR}$ como bases de datos confiables, actuales, con accesibilidad a documentos y permiten identificar las citaciones, por lo cual su uso en la literatura es frecuente (Hall, 2011). Para localizar los trabajos relacionados y recuperar la información disponible de tesis, informes, documentos oficiales y artículos científicos se aplicó la siguiente ecuación de búsqueda para los campos título, resumen y/o palabras claves: (fintech) OR (financial inclusion) AND (covid*) OR (pandemic) OR (*crisis). Los resultados fueron priorizados a partir del número de citaciones y la relevancia de búsqueda durante el año 2020; luego se procede a identificar el alcance y el direccionamiento del documento hacia lo reflexivo o lo propositivo.

\section{RESULTADOS}

A pesar de que este artículo es anticipatorio y busca esbozar la escasa investigación existentes sobre la pandemia y las proyecciones de inclusión financiera como estrategia de recuperación, es posible destacar artículos académicos y publicaciones que además de evaluar impactos de las pandemias y crisis anteriores, presentan pronósticos acerca de cómo la COVID-19 generará consecuencias con relación a las afectaciones socioeconómicas, de mercados y de instituciones financieras. Dichas 
apreciaciones están enfocadas hacia aprendizajes previos, modelación, aplicación de las finanzas digitales, educación financiera y recomendaciones para la recuperación económica efectiva.

A partir de la ecuación de búsqueda, se rescatan 76 resultados, los cuales no logran construir indicadores de calidad, cantidad o estructura como los propone Wallin (2005), una vez que la muestra es pequeña y aún no se tienen acumulación de datos o distribuciones evidentes de relevancia en autores o textos al año 2020. Sin embargo, se logran rescatar los principales documentos de acuerdo con la relevancia de sus autores y al número de lecturas para construir la tabla 1: resumen sobre los principales documentos y autores analizados a partir de una revisión de sus contenidos y alcances.

Tabla 1. Artículos relevantes sobre inclusión financiera como estrategia de recuperación.

\begin{tabular}{|c|c|c|}
\hline Autor & Título del Artículo & Principales Aportes \\
\hline $\begin{array}{l}\text { (Wójcik y } \\
\text { Loannou, } \\
2020 \text { ) }\end{array}$ & $\begin{array}{l}\text { COVID-19 and } \\
\text { Finance: Market } \\
\text { Developments So } \\
\text { Far and Potential } \\
\text { Impacts on the } \\
\text { Financial Sector } \\
\text { and Centers }\end{array}$ & $\begin{array}{l}\text { Documento de carácter reflexivo. Se presenta un } \\
\text { estudio sobre los impactos de la crisis en las economías } \\
\text { y especialmente en los mercados de capitales a nivel } \\
\text { global, con lo cual refieren la consolidación de los } \\
\text { servicios financieros relacionados con la virtualidad } \\
\text { y el no contacto físico, por lo cual se prevé una } \\
\text { aceleración en la aplicación de nuevas tecnologías } \\
\text { financieras generando desafíos para la banca tradicional } \\
\text { y estableciendo necesidades de mayor inclusión ante } \\
\text { la posibilidad de fuga de clientes por miedo a los } \\
\text { contactos físicos de la banca tradicional. }\end{array}$ \\
\hline $\begin{array}{l}\text { (Nizam et } \\
\text { al., 2020) }\end{array}$ & $\begin{array}{l}\text { Financial } \\
\text { inclusiveness and } \\
\text { economic growth: } \\
\text { new evidence } \\
\text { using a threshold } \\
\text { regression analysis }\end{array}$ & $\begin{array}{l}\text { Documento de carácter cuantitativo. Se investiga el } \\
\text { efecto de la inclusión financiera en el crecimiento } \\
\text { económico de determinados países desarrollados y } \\
\text { en desarrollo en los años } 2014 \text { y } 2017 \text { y se reflexiona } \\
\text { sobre la proyección a } 2020 \text { dada la situación } \\
\text { coviD-19. El papel de la inclusividad financiera en } \\
\text { el crecimiento económico se estima posteriormente } \\
\text { mediante una técnica de regresión de umbral } \\
\text { transversal, concluyendo que existe un efecto umbral } \\
\text { del nexo entre la inclusión financiera y el crecimiento, } \\
\text { lo que significa que la inclusión financiera muestra } \\
\text { una relación positiva no monótona con el crecimiento } \\
\text { económico. }\end{array}$ \\
\hline
\end{tabular}




\begin{tabular}{|c|c|c|}
\hline Autor & Título del Artículo & Principales Aportes \\
\hline $\begin{array}{l}\text { (Cavoli et } \\
\text { al., 2020) }\end{array}$ & $\begin{array}{l}\text { Does Financial } \\
\text { Inclusion Amplify } \\
\text { Output Volatility } \\
\text { in Emerging } \\
\text { and Developing } \\
\text { Economies? }\end{array}$ & $\begin{array}{l}\text { Documento reflexivo con experimentación empírica. } \\
\text { En este documento se presenta un análisis sobre la } \\
\text { inclusión financiera como factor de volatilidad de } \\
\text { la producción, lo cual es motivo de preocupación } \\
\text { para los responsables de la política monetaria de } \\
\text { las economías emergentes y en desarrollo (EME) } \\
\text { especialmente en épocas de crisis. La estimación } \\
\text { empírica encuentra un equilibrio fuerte y persistente } \\
\text { entre una mayor inclusión financiera y la estabilidad } \\
\text { del producto. En el documento también se encuentran } \\
\text { pruebas sólidas y contundentes de la no linealidad } \\
\text { que rige esta relación. Los países con altos grados de } \\
\text { inclusión financiera, así como aquellos con ingresos } \\
\text { relativamente más bajos, tienden a experimentar una } \\
\text { importante compensación entre la inclusión financiera } \\
\text { y la estabilidad del producto. }\end{array}$ \\
\hline $\begin{array}{l}\text { (Sahay et } \\
\text { al., 2020) }\end{array}$ & $\begin{array}{l}\text { The Promise of } \\
\text { Fintech: Financial } \\
\text { Inclusion in the } \\
\text { Post COVID-19 Era }\end{array}$ & $\begin{array}{l}\text { Documento de carácter reflexivo donde se } \\
\text { presenta un análisis del cambio de la banca y de } \\
\text { la transformación de esta durante los últimos } \\
\text { años. Adicionalmente describe la pandemia de la } \\
\text { COVID-19, como el momento para que se acelere } \\
\text { y mejore la inclusión financiera, en medio del } \\
\text { distanciamiento social y las medidas de contención. } \\
\text { Además, propone premisas sobre implementación de } \\
\text { educación financiera. }\end{array}$ \\
\hline $\begin{array}{l}\text { (Goodell, } \\
\text { 2020) }\end{array}$ & $\begin{array}{l}\text { COVID-19 and } \\
\text { finance: Agendas } \\
\text { for future research }\end{array}$ & $\begin{array}{l}\text { Documento de carácter reflexivo sobre el impacto } \\
\text { económico y social de la COVID-19 y se presentan } \\
\text { posibles implicaciones e impactos en los mercados e } \\
\text { instituciones financieras, basado en anteriores crisis. }\end{array}$ \\
\hline $\begin{array}{l}\text { (Lusardi et } \\
\text { al., 2020) }\end{array}$ & $\begin{array}{l}\text { Building up } \\
\text { financial literacy } \\
\text { and financial } \\
\text { resilience }\end{array}$ & $\begin{array}{l}\text { Artículo de carácter mixto. A partir del índice de } \\
\text { finanzas personales (P-Fin) del Instituto TIAA-GFLEC } \\
\text { de } 2020 \text { se establecen niveles de fragilidad en } \\
\text { Estados Unidos mucho antes de que la pandemia } \\
\text { COVID-19 golpeara su economía, especialmente en } \\
\text { afroamericanos y personas de ingresos bajos y la } \\
\text { relaciona con el nivel de educación financiera. }\end{array}$ \\
\hline
\end{tabular}

Fuente: Construcción propia a partir de la base de datos Scopus.

A partir de estos documentos y con apoyo en otros autores se destacan los siguientes aspectos claves con relación a los aprendizajes anteriores, la importancia creciente de las finanzas digitales, la educación financiera como estrategia de inclusión y, finalmente, propuestas para actuar frente a la situación post-COVID-19. 


\subsection{Aprendizajes de anteriores crisis}

El impacto de pandemias y crisis económicas globales en el pasado, han dejado algunas enseñanzas que son rescatadas hoy y que se pretenden retomar como herramientas de apoyo y soporte de recuperación y crecimiento. Lagoarde-Segot y Leoni (2013) y Demir et al. (2020) señalan que es claro que los bancos por su naturaleza, son directamente vulnerables a las crisis, por la probabilidad de que se produzcan préstamos improductivos y desaceleración del consumo, por lo cual dichas entidades se han blindado con normativas sobre la titularización de créditos, endurecimiento de políticas sobre apalancamiento y reducción de la financiación a corto plazo del mercado de capitales, además de la posibilidad de modelar mejor y de manera más dinámica su información financiera y de clientes a través de los procesos Fintech (Stoeckli et al., 2018; Valencia et al., 2017).

Por otro lado, se tiene claro que los grandes perdedores, son las comunidades, la sociedad en general, una vez que gran parte de lo que mueven las entidades financieras corresponden a microfinanciación a los pobres, quienes se verán presionados durante una conmoción global (Skoufias, 2003).

Las anteriores crisis también han puesto sobre la mesa la previsión, en la economía global, de la afectación de situaciones como una pandemia; sobre sí la COVID-19 debe ser considerado un evento imprevisible de consecuencias extremas. Autores como Tamura y Sawada (2011) y Cavallo et al. (2013) hablan de la posibilidad de que tales eventos sean asegurables y que por supuesto, este tipo de seguros solo estaría disponible para quienes estén financieramente incluidos, aportando generación de garantías que soporten una rápida recuperación. En episodios anteriores como catástrofes naturales y crisis sanitarias, las personas que no tienen seguros, o medios de garantías financiera logran superar la crisis en los primeros cinco años (Hannig y Jansen, 2011).

Lagoarde-Segot y Leoni (2013) y Fisch et al. (2019) afirman que para minimizar las posibilidades de futuras crisis, los gobiernos y los reguladores bancarios deberían centrarse en mejorar el aprendizaje, la creación de conocimientos y la comprensión de la banca y de las finanzas en general, pues el mal generalizado que ha mantenido y ahondado las crisis está relacionado con el bajo entendimiento del sistema financiero y la poca planeación de los ciudadanos, quienes al final de cuentas son los que más sufren en las crisis.

La financiación de las empresas y el costo de capital también es un tema de aprendizaje de anteriores situaciones, pues luego de episodios de crisis se generan repercusiones negativas en la demanda interna mundial, lo que supone que los 
mercados financieros sufran desvalorización en sus acciones y pasen a tener en cuenta las potencialidades de los eventos perjudiciales a futuro, y así generar un impacto a largo plazo en las primas de riesgo (Al-Awadhi et al., 2020; Goodell, 2020). Es por esto que dentro de la inclusión financiera también se habla de las Pymes, quienes deben estar a la vanguardia en estrategias financieras para lograr reponerse y sobrevivir a las crisis y situaciones que interrumpan su funcionamiento (Igual Molina, 2019).

\subsection{Finanzas digitales}

Sahay et al. (2020) en colaboración con el Fondo Monetario Internacional presentan un estudio en el cual afirman que en una muestra de 52 países y en una ventana de observación de 2014 a 2017, las finanzas digitales logran aumentar la inclusión financiera, incluso en lugares de África y Asia donde la penetración bancaria tradicional no presenta crecimiento, una vez que la inclusión financiera digital logra desarrollarse con herramientas tan simples como un celular con acceso a mensajes de texto. De este estudio presentado en 2020, se destacan varias premisas (ver tabla 2) que se tienen en cuenta sobre la evolución de la temática y los pronósticos y consideraciones a ser tenidas en cuenta en este momento, en el cual toma más relevancia el tema de la digitalización.

Tabla 2. Premisas Finanzas Digitales.

\begin{tabular}{ll}
\hline \multicolumn{1}{c}{ Premisa } & \multicolumn{1}{c}{ Consideración } \\
\hline Las finanzas digitales & La inclusión financiera logra desarrollarse y adaptarse a \\
aumentan la inclusión & herramientas tecnológicas tan diversas como el internet \\
financiera, incluso & o un celular con mensajes de texto. Esto hace que en \\
en lugares donde la & lugares donde no se registra crecimientos bancarios \\
penetración bancaria & como África y Asia se logre inclusión de nuevas personas \\
tradicional está estancada. & al sistema financiero.
\end{tabular}


Revisión bibliográfica sobre inclusión financiera como estrategia de recuperación y de crecimiento fintech

\section{Premisa}

La inclusión financiera digital se asocia con un mayor crecimiento del PIB.
Las Fintech están contribuyendo a cerrar brechas de género.

La prestación de servicios financieros digitales continúa en evolución.

La seguridad de la inclusión financiera digital.

Las finanzas digitales pueden crear nuevos riesgos para la inclusión financiera.
Consideración

La adopción de pagos digitales está asociada de manera significativa y positiva con crecimiento en los países, consistente con la noción de crecimiento causado por el desarrollo Fintech. Esto señala que el crecimiento luego de la pandemia de COVID-19 tendrá incidencia de las Fintech y que estas apoyarán en la recuperación, ya que los países con mayor inclusión financiera digital tienen mayor facilidad para asegurar continuidad en el acceso, seguridad y ofrecimiento de los servicios financieros (en especial el crédito) y se podrá mantener consumo y la innovación de la producción a través de la economía digital. Sin embargo, si las pequeñas compañías Fintech (que son las que más llegan a hogares de bajos ingresos) se ven afectadas por la crisis y desaparecen, podría aumentar la brecha entre ricos y pobres.

Aunque la tecnología en la actualidad genera mayor acercamiento de las mujeres con el sistema financiero aún existen barreras sociales, culturales, de seguridad y de disparidad en el acceso a los recursos.

El desarrollo de la modelación y la accesibilidad del Internet, están permitiendo que los servicios financieros no solo dependan de bancos sino también de nuevas entidades que aparecen como competencia y/o complemento a la banca tradicional. La COVID-19 puede ser una dura prueba para estos mercados emergentes que no tienen el mismo apoyo que recibe el sector bancario

En etapas en las cuales se acaba de pasar por una crisis, es fundamental que los desarrollos incluyan seguridad y regulación que conduzcan a estabilidad y credibilidad.

Si bien, la inclusión financiera se relaciona como estrategia de crecimiento y apoyo a las Fintech, existen riesgos derivados de las desigualdades en la infraestructura digital de la personas y comunidades, además de la gran cantidad de restricciones con relación a la alfabetización financiera y el manejo de datos y analítica. Aunque el modelo plantea accesos sencillos y en el ámbito tecnológico, es importante hacer un seguimiento a la brecha tecnológica y la especialización de los productos financieros.

Fuente: Construcción propia a partir de Sahay et al. ( 2020) y Li et al. (2020). 
Las finanzas digitales han ganado y continuarán ganando espacio en los mercados financieros, con la creación de nuevos y estructurados productos que buscan a través de las posibilidades de la cuarta revolución, generar soluciones a la medida de las necesidades de los clientes. Sin embargo, como se indica en las premisas, no se puede dejar de lado que su propósito no debe ser exclusivo de intensiones capitalistas, sino que también debe estar amarrado al desarrollo y alineado con los ODS para buscar generalizar servicios y generar soluciones sostenibles y sustentables en el tiempo.

\subsection{Educación Financiera como herramienta de inclusión.}

La educación o alfabetización financiera son herramientas fundamentales para el ahorro, no solo a largo, sino también a corto plazo; como los llamados ahorros de precaución que aumentan la capacidad de una inversión frente a una crisis financiera. Quienes tienen más conocimientos financieros tienen más probabilidades de conseguir dinero si se produce una crisis inesperada y menos probabilidad de depender de préstamos (Fisch et al., 2019).

En situaciones adversas, como las que se presentan en un escenario pandémico que llega a afectar la economía, los conocimientos sobre tipología de crédito y gestión de deudas, que por lo general están relacionados con pagos altos de intereses, comisiones y superación de límites establecidos para pagos; son fundamentales y se entienden como ventajas y elementos de resiliencia que generan una reducción en el plazo estimado de la recuperación (Lusardi y Tufano, 2015).

El Centro Global para la Excelencia en Educación Financiera, GFLEC (por sus siglas en inglés) y la Asociación Americana de Seguros y Rentas en América, TIAA (por sus siglas en inglés), desarrollan el índice P-Fin que mide la alfabetización financiera en los Estados Unidos con relación a las ganancias, el consumo, el ahorro, la inversión, los préstamos, la administración de la deuda, los seguros, la comprensión de riesgos y el acudir a las fuentes de información, como dimensiones fundamentales de la educación financiera (Lusardi et al., 2020). Este índice muestra que, aunque en 2020 las dimensiones analizadas fueron superiores al 2017, los valores en general aún son bastante bajos, lo que representa notas reprobables, lo que señala que desde antes de la pandemia la población ya era vulnerable desde el punto de vista financiero, incluso en mejores condiciones socioeconómicas como se muestra en la figura 3. En lo relacionado con la comprensión de riesgos asociados con adquisición y gestión de productos financieros, se muestra el menor número de aciertos por parte de los encuestados, con $39 \%$ de respuestas correctas en 2020, una leve mejoría con relación a los datos de 2017, frente a preguntas como ¿̇abe que 
es una tasa de mora?, o ¿̇abe cuál es su perfil de riesgo?, que de manera genérica buscan identificar su acercamiento y entendimiento de los productos financieros generalizados como tarjetas débito y créditos.

Figura 3. Respuestas acertadas a cuestionamientos sobre las dimensiones de la Educación Financiera.



$2020 \quad \square 2017$

Fuente: (TIAA, 2020)

De acuerdo con Lusardi et al. (2020), en momentos de agitación económica el riesgo y la incertidumbre se amplifican y generan mayores dificultades, en una mayor proporción en mujeres, personas de bajos ingresos, desempleados, discapacitados, jóvenes y personas menos ocupadas; asiéndose fundamental que en procesos de recuperación se tengan en cuenta dentro de las políticas institucionales, la educación financiera como elemento aportante a la reconstrucción y fortalecimiento del flujo económico y del tejido financiero.

Los conocimientos financieros permiten planeación para el futuro y requieren ser diversos e incluir aspectos débiles como el enfoque en riesgos, los seguros y las inversiones como aspectos relevantes en la interacción con servicios Fintech (Panos y Wilson, 2020).

\subsection{Propuestas para promover la inclusión financiera post covid-19.}

India, uno de los países más afectados por la COVID-19 ha presentado su Estrategia Nacional para la Educación Financiera 2020-2025 (The Economic Times, 2020). En este plan expone la necesidad de educación e inclusión financiera como herramientas de desarrollo y extiende la participación de niños y jóvenes en edad escolar y 
adultos, para empoderar a toda la comunidad a tomar decisiones informadas con estrategias como:

- Contenidos de alfabetización financiera para estudiantes, maestros, adultos, jóvenes, nuevos empresarios y trabajadores de Pymes.

- Capacitaciones previas al acceso de productos financieros y enfoque en capacidades de intermediarios.

- Elaboración de un código de conducta para los proveedores de educación financiera y para los proveedores de servicios financieros.

- Integrar el contenido de la educación financiera en el programa de estudios de las escuelas y en diversos cursos profesionales y de capacitación en niveles de educación superior.

Por su parte, el Banco Mundial (2020), recomienda a los países desarrollar un plan que incluya una adecuada planeación, regulación, seguridad cibernética e inserción de actores al sistema Fintech a través de la inclusión financiera. La rápida inclusión sin una reglamentación adecuada y sin conocimientos financieros puede dar lugar a una inestabilidad financiera debido a prácticas inapropiadas de las instituciones que pondrían en peligro la confianza de los nuevos usuarios (Ozili y Arun, 2020).

El Fondo Monetario Internacional, muestra que las finanzas digitales aumentan la inclusión financiera y están asociadas con un mayor crecimiento del PIB al señalar que la inclusión financiera podría desempeñar un papel fundamental en la mitigación del impacto económico y social de la actual crisis de la COVID-19. La ampliación del acceso financiero de los hogares de bajos ingresos y las pequeñas empresas también podría apoyar una recuperación más inclusiva. Sin embargo, estas posibilidades no pueden darse por sentadas, ya que la pandemia podría acelerar los riesgos preexistentes de exclusión financiera y dar lugar a nuevos riesgos para el propio sector de la tecnología financiera (Brown et al., 2020; Sahay et al. 2020).

\section{CONCLUSIONES Y DISCUSIONES}

La inclusión financiera es una estrategia que tiene implicaciones en la reducción de la pobreza, pero no es la única frente a ese fin, el cual debe ser atendido de manera conjunta del sector público y privado. 
El sector público está llamado a generar políticas eficientes que incentiven a las entidades financieras a desarrollar una inclusión financiera basada y orientada en la ampliación de la red y en sus beneficios como usuarios y como participes de un sistema sostenible. Por su parte, el sector privado debe pensar más en la expansión, en la vinculación y en la estabilidad a largo plazo de todo el sistema, con la incorporación de alternativas de participación para las personas de menos recursos.

La tecnología transforma el panorama del sector financiero, siendo responsable de un mayor acceso a los servicios financieros de manera profunda. Estos cambios han estado en movimiento durante varios años, con afectaciones en casi todos los países del mundo de manera gradual. Durante la pandemia de la COVID-19, la tecnología ha creado nuevas oportunidades para que los servicios financieros digitales aceleren y mejoren la inclusión financiera, en medio del distanciamiento social y las medidas de contención, en gran parte a su característica de no contacto físico y transacciones virtuales. Al mismo tiempo, los riesgos que surgen antes de la COVID -19, a medida que los servicios financieros digitales se desarrollaban, se están volviendo aún más relevantes.

Sin embargo, como lo expone Ozilia y Arun (2020) también es importante tener en cuenta que la inclusión financiera y las Fintech pueden generar efectos negativos cuando los proveedores de servicios digitales buscan el lucro y la rentabilidad, con el desarrollo de estrategias agresivas de persuasión que en vez de generar beneficios de conectividad para las personas, creen costos y mayor desconfianza del sistema financiero y de las plataformas, aumentando la segregación de los clientes, pues es bien sabido que los clientes de ingresos bajos y pobres tienen menores rendimientos que los clientes de ingresos altos.

Las finanzas digitales pueden crear nuevos riesgos para la inclusión financiera que se derivan del acceso desigual a la infraestructura digital, las limitaciones a la alfabetización financiera y digital, y los posibles sesgos amplificados por las nuevas fuentes de datos y el análisis de datos (Sahay et al., 2020).

Finalmente, se hace importante rescatar el papel de la educación financiera como elemento de inclusión, una vez que desde los esfuerzos para promover la bancarización se muestra que la capacitación en temas financieros genera procesos de empoderamiento en las personas y en sus comunidades, además de generación de tejido financiero y disminución de la vulnerabilidad con relación a posibles crisis al contar con herramientas para la toma de decisiones informadas. 


\section{BIBLIOGRAFÍA}

Akhtaruzzaman, M., Boubaker, S. y Sensoy, A. (2020). Financial contagion during COVID-19 crisis. Finance Research Letters, 34, 1-20. https://doi.org/10.1016/j.frl.2020.101604

Al-Awadhi, A. M., Alsaifi, K., Al-Awadhi, A. y Alhammadi, S. (2020). Death and contagious infectious diseases: Impact of the COVID-19 virus on stock market returns. Journal of Behavioral and Experimental Finance, 27, 1-5. https://doi.org/10.1016/j.jbef.2020.100326

Arner, D. W., Buckley, R. P., Zetzsche, D. A. y Veidt, R. (2020). Sustainability, FinTech and Financial Inclusion. European Business Organization Law Review, 21 (1), 1-5. https://doi.org/10.1007/ s40804-020-00183-y

Ashraf, B. N. (2020). Stock markets' reaction to COVID-19: Cases or fatalities? Research in International Business and Finance, 54 (101249), 1-7. https://doi.org/10.1016/j.ribaf.2020.101249

Behravesh, N. y Rocha, E. W. (2020). Interim Global Economic Forecast. IHS Markit. http://www. oecd.org/economic-outlook/

Brown, R., Rocha, A. y Cowling, M. (2020). Financing entrepreneurship in times of crisis: Exploring the impact of COVID-19 on the market for entrepreneurial finance in the United Kingdom. International Small Business Journal: Researching Entrepreneurship, 38 (5), 380-390. https://doi. org/10.1177/0266242620937464

Bull, T., Chen, S. y Chiselita, D. (2019). Global FinTech Adoption Index 2019. Ernst E Young. https:// www.ey.com/en_gl/ey-global-fintech-adoption-index

Cavallo, E., Galiani, S., Noy, I. y Pantano, J. (2013). Catastrophic natural disasters and economic growth. Review of Economics and Statistics, 95 (5),1549-1561. https://doi.org/10.1162/REST_a_00413

Cavoli, T., Gopalan, S. y Rajan, R. S. (2020). Does Financial Inclusion Amplify Output Volatility in Emerging and Developing Economies? Open Economies Review, 31 (4), 901-930. https://doi. org/10.1007/s1 1079-019-09568-0

Chattopadhyay, S. K. (2011). Financial Inclusion in India: A Case Study of West Bengal. Reserve Bank of India Working Paper, 2 (8), 1 42. https://doi.org/10.1007/s1 1079-019-09568-0

Demir, A., Pesqué-Cela, V., Altunbas, Y. y Murinde, V. (2020). Fintech, financial inclusion and income inequality: a quantile regression approach. European Journal of Finance, 26 (4) 297-301. https://doi.org/10.1080/1351847X.2020.1772335

Díaz, H., Sosa, M., y Ortiz, E. (2018). Inclusión Financiera y ahorro en México: un análisis logístico binario y de redes neuronales artificiales. Estocástica: Finanzas y Riesgo, 8 (1), 53-84. https:// doi.org/2007-5375

Dorfleitner, G., Hornuf, L., Schmitt, M. y Weber, M. (2017). The FinTech market in Germany. FinTech in Germany. Springer. https://doi.org/10.1007/978-3-319-54666-7

Fisch, J. E., Lusardi, A. y Hasler, A. (2019). Defined Contribution Plans and the Challenge of Financial Illiteracy. SSRN Electronic Journal, 103 (3), 19-22 https://doi.org/10.2139/ssrn.3384778

G20 (2011). G20 Principles for Innovative Financial Inclusion. Alliance for Financial Inclusion. https:// sustainabledevelopment.un.org/index.php?page $=$ view\&type $=400 \xi \mathrm{nr}=2658 \mathrm{E} \mathrm{menu}=35$ 
\#: : text=The\%20G20\%20Principles\%20for\%20Innovative\%20Financial\%20Inclusion\%20 consist\%20of\%20nine,G20's\%20Financial\%20Inclusion\%20Action\%20Plan.

Gabor, D. y Brooks, S. (2017). The digital revolution in financial inclusion: international development in the fintech era. New Political Economy, 22 (4), 423-436. https://doi.org/10.1080/13 563467.2017 .1259298

Goodell, J. W. (2020). COVID-19 and finance: Agendas for future research. Finance Research Letters, 35, 1-5. https://doi.org/10.1016/j.frl.2020.101512

GPFI (2011). G20 Financial Inclusion Action Plan. Global Partnership for Financial Inclusion. https:// doi.org/10.1007/s1 1079-019-09568-0

Hall, M. C. (2011). Publish and perish? Bibliometric analysis, journal ranking and the assessment of research quality in tourism. Tourism Management, 32 (1), 16-27. https://doi.org/10.1016/j. tourman.2010.07.001

Hannig, A. y Jansen, S. (2011). Financial inclusion and financial stability: Current policy issues. Financial Market Regulation and Reforms in Emerging Markets, 1 (259), 1-34. https://doi.org/10.2139/ ssrn. 1729122

Igual Molina, D. (2019). Las fintech y la reinvención de las finanzas. Oikonomics, 10, 22-44. https:// doi.org/10.7238/o.n10.1811

International Monetary Fund (2014). World Economic Outlook: Legacies, Clouds, Uncertainties, vol.3. https://www.imf.org/en/Publications/WEO/Issues/2016/12/31/Legacies-Clouds-Uncertainties

Lagoarde-Segot, T. y Leoni, P. L. (2013). Pandemics of the poor and banking stability. Journal of Banking and Finance, 37 (11), 4574-4583. https://doi.org/10.1016/j.jbankfin.2013.04.004

Li, J., Wu, Y. y Xiao, J. J. (2020). The impact of digital finance on household consumption: Evidence from China. Economic Modelling, 86, 317-326. https://doi.org/10.1016/j.econmod.2019.09.027

Lusardi, A. y Tufano, P. (2015). Debt literacy, financial experiences, and overindebtedness. Journal of Pension Economics and Finance, 14 (4), 332-368. https://doi.org/10.1017/S1474747215000232

Lusardi, A., Hasler, A. y Yakoboski, P. J. (2020). Building up financial literacy and financial resilience. Mind and Society, 19 (1), 1-7. https://doi.org/10.1007/s1 1299-020-00246-0

Nizam, R., Karim, Z. A., Rahman, A. A. y Sarmidi, T. (2020). Financial inclusiveness and economic growth: new evidence using a threshold regression analysis. Economic Research-Ekonomska Istrazivanja, 33 (1), 1465-1484. https://doi.org/10.1080/1331677X.2020.1748508

Ozili, P. K. (2018). Impact of digital finance on financial inclusion and stability. Borsa Istanbul Review, 18 (4), 329-340. https://doi.org/10.1016/j.bir.2017.12.003

Ozili, P. K., y Arun, T. (2020). Spillover of COVID-19: Impact on the Global Economy. SSRN Electronic Journal, 1, 1-17. https://doi.org/10.2139/ssrn.3562570

Panos, G. A. y Wilson, J. O. S. (2020). Financial literacy and responsible finance in the FinTech era: capabilities and challenges. European Journal of Finance, 26 (4), 297-301. https://doi.org/ 10.1080/1351847X.2020.1717569 
Philippon, T. y Philippon, T. (2019). The FinTech Opportunity. National Bureau of Economic Research. https://doi.org/10.3386/w22476

Raccanello, K., Raccanello, K., y Herrera Guzmán, E. (2014). Educación e inclusión financiera. Revista Latinoamericana de Estudios Educativos, 44 (2), 119-141. https://www.redalyc.org/articulo. oa?id $=270 / 27031268005$

Rizwan, M. S., Ahmad, G. y Ashraf, D. (2020). Systemic risk: The impact of COVID-19. Finance Research Letters, 36, 101682. https://doi.org/10.1016/j.frl.2020.101682

Romanova, I., y Kudinska, M. (2016). Banking and fintech: A challenge or opportunity? Contemporary Studies in Economic and Financial Analysis, 98, 21-35. https://doi.org/10.1108/S1569375920160000098002

Sahay, R., Allmen, U. E. von, Lahreche, A., Khera, P., Ogawa, S., Bazarbash, M., y Beaton, K. (2020). The Promise of Fintech: Financial Inclusion in the Post COVID-19 Era. IMF Departmental Papers / Policy Papers. 09. https://ideas.repec.org/p/imf/imfdep/20-09.html

Skoufias, E. (2003). Economic crises and natural disasters: Coping strategies and policy implications. World Development, 31 (7), 1087-1102. https://doi.org/10.1016/S0305-750X(03)00069-X

Stoeckli, E., Dremel, C. y Uebernickel, F. (2018). Exploring characteristics and transformational capabilities of InsurTech innovations to understand insurance value creation in a digital world. Electronic Markets. https://doi.org/10.1007/s1 2525-018-0304-7

Tamura, S. y Sawada, Y. (2011). Consumption Insurance against Unforeseen Epidemics: The case of avian influenza in Vietnam. RIETI Discussion Paper Series 09-E-023 n 09023. https://core. ac.uk/download/pdf/6957028.pdf

The Economic Times (2020). Reserve Bank of India suggests a five-point action plan to promote financial education. India Times. https://economictimes.indiatimes.com/news/economy/policy/ reserve-bank-of-india-suggests-a-five-point-action-plan-to-promote-financial-education/ articleshow/77653820.cms?utm_source=contentofinterestEutm_medium =textEutm_ campaign $=$ cppst

The World Bank (2018). UFA2020 Overview: Universal Financial Access by 2020. https://www.worldbank. org/en/topic/financialinclusion/brief/achieving-universal-financial-access-by-2020

TIAA, G. (2020). The 2020 TIAA Institute-GFLEC Personal Finance Index. https://gflec.org/initiatives/ personal-finance-index/

United Nations (2014). Impact of access to financial services, including by highlighting remittances on development: Economic empowerment of women and youth. Trade and Development Commission Expert Meeting on the Impact of Access to Financial Services, Including by Highlighting Remittances on Development: Economic Empowerment of Women and Youth. https://unctad.org/system/ files/official-document/ciem6d2_en.pdf

United Nations (2020). UN launches COVID-19 plan that could 'defeat the virus and build a better world'. Prevention and Control. https://doi.org/10.1016/j.precon.2005.06.007

United Nations (2020). Shared responsibility, global solidarity: responding to the socio-economic impacts of COVID-19. https://unsdg.un.org/sites/default/files/2020-03/SG-Report-Socio-Economic-Impact-of-Covid19.pdf 
Valencia, D. C., Arevalo, J. B., y Rodríguez, D. (2017). Análisis bibliométrico sobre direccionamiento de los estudios en Riesgos Financieros. Revista Venezolana de Gerencia, 38 (59), 2-13. http:// www.revistaespacios.com/al7v38n59/17385902.html

Venkatesh, V., Thong, J. Y. L., Statistics, B., Xu, X. y Acceptance, T. (2016). Unified Theory of Acceptance and Use of Technology: A Synthesis and the Road Ahead. Journal of the Association for Information Systems, 17 (5), 328-376. https://doi.org/10.1080/1097198X.2010.10856507

Villa, E., Cardona Valencia, D., Valencia-Arias, A., Hormechea, K., y García, J. (2020). Transformative Innovation Policy, SDGs, and the Colombian University. Springer. https://doi.org/10.1007/978-3030-26157-3_14

Wallin, J. A. (2005). Bibliometric methods: Pitfalls and possibilities. Basic and Clinical Pharmacology and Toxicology, 97 (5), 261-275 https://doi.org/10.1111/j.1742-7843.2005.pto_139.x

Wójcik, D., y Loannou, S. (2020). COVID-19 and Finance: Market Developments So Far and Potential Impacts on the Financial Sector and Centres. Tijdschrift Voor Economische En Sociale Geografie, 111 (3), 387-400. https://doi.org/10.1111/tesg. 12434

World Bank (2020). 2020 Global Economic Prospects: June 2020. In The Financial Crisis and the Global South. https://doi.org/10.2307/j.ctt183pb3w.5

Zhang, D., Hu, M., y Ji, Q. (2020). Financial markets under the global pandemic of COVID-19. Finance Research Letters, 36, 1-6. https://doi.org/10.1016/j.frl.2020.101528 